\title{
Original
}

\section{Relationship between plasma level and therapeutic effect of sulpiride}

\author{
Satoru Shima \\ Department of Neuropsychiatry, School of Medicine, Keio University \\ 35 Shinanomachi, Shinjuku-ku, Tokyo 160, Japan
}

(Received for publication on July 29, 1986)

\begin{abstract}
Fifty-six depressed patients were administered sulpiride. No significant correlation was found between plasma levels of sulpiride and clinical responses. Basal 3-methoxy-4-hydroxyphenylglycol and dopamine- $\beta$-hydroxylase levels failed to predict the therapeutic effect.
\end{abstract}

Key words: sulpiride, therapeutic effect, dopamine- $\beta$-hydroxylase, 3-methoxy4-hydroxyphenylglycol

\section{Introduction}

Sulpiride, one of substituted benzamide compounds developed by Besancon in the 1960s, has been extensively used as an unique psychotropic agent in Japan and other countries. Sulpiride has a disinhibitory as well as an antipsychotic action. This dual effect depends on doses; at low doses (150-300 mgs/day) sulpiride shows the disinhibitory effect and at high doses (600-2,000 mgs/day) antipsychotic effect. Jenner and Marsden ${ }^{1}$ have hypothesized that at low doses sulpiride possesses a preferential pre-synaptic action that leads to dopamine hyperfunction and increased psychomotility, while at high doses it acts mainly as post-synaptic dopamine antagonist similar to other neuroleptics.

Relationship between blood levels of antidepressants and clinical efficacy is one of major concerns in the field of modern clinical psychopharmacology. While reports of each investigator are not always consistent and no confirmed results have been obtained for any antidepressants, an inverted $U$ wave correlation for nortriptyline and a positive

島悟 
linear correlation for imipramine are supposed to exist by some researchers. To date, only two studies were reported regarding sulpiride, in which no significant correlations were found between its blood levels and clinical effect.:.3 The sample sizes were, however, too small to draw a conclusion in these two studies; 14 and 11 respectively.

3-methoxy-4-hydroxyphenylglycol (MHPG) is thought to be the major central metabolite of noradrenaline. There has recently ample evidence that basal (pre-treatment) MHPG level relates to clinical response to tricyclic antidepressants. ${ }^{4,5}$ Dopamine$\beta$-hydroxylase (DBH), the enzyme which synthesizes noradrenaline from dopamine, is another marker of noradrenaline activity. The results of studies of the enzyme in depressed patients are variable.

The present study aimed at investigating the relationship of the plasma levels of sulpiride with its clinical effects in a larger sample and at clarifying the relevancy of plasma MHPG and DBH levels to the therapeutic effects.

\section{Subjects and methods}

Seventy-five depressed patients who met the following criteria were studied: (1) major depression by the criteria of DSM III, (2) no serious medical illnesses, (3) no previous psychiatric treatment within at least 4 weeks.

Patients were randomly given $50 \mathrm{mg}$ or $100 \mathrm{mg}$ sulpiride orally three times per day during four weeks. Severity of depressed state was assessed weekly by the Hamilton Rating Scale for Depression (HRSD). Clinical response was defined as a proportion of basal HRSD to the final HRSD total score. The global improvement rating (GIR) was also utilized to estimate therapeutic effect. New Castle Scale ${ }^{6}$ was also used to estimate endogenuity of depression.

Basal MHPG, DBH and prolactin samples were taken in the morning prior to medication. Blood samples for the determination of plasma sulpiride concentration were taken before the morning dose after one to three weeks treatment, since, as shown in Bjerkenstedt's study, ${ }^{7}$ the blood sulpiride concentration is thought to reach the steady state within one week treatment. Plasma MHPG, DBH and prolactin levels were also analyzed for the second sample. Plasma sulpiride concentration was determined using high-performance liquid chromatographic technique (HPLC). Plasma MHPG level was also analyzed by HPLC. 8 Plasma DBH activity was assayed by the spectrophotometric method of Nagatsu and Udenfriend. ${ }^{9}$ Plasma prolactin level was determined by radioimmunoassay.

All the patients gave informed consent prior to the investigation.

\section{Results}

Of 75 original sample, 19 patients were excluded; one patient was refered to a 
Table 1

Plasma levels of MHPG, DBH, prolactin and sulpiride

\begin{tabular}{lcc} 
& Pre-treatment & Post-treatment \\
MHPG (ng/ml) & $5.5 \pm 1.7$ & $5.1 \pm 1.2$ \\
DBH (IU/L) & $31.5 \pm 16.9$ & $30.5 \pm 14.4$ \\
Prolactin (ng/ml) & $10.3 \pm 5.3$ & $112.8 \pm 87.2$ \\
Sulpiride (ng/ml) & & $259 \pm 210$ \\
\hline
\end{tabular}

mental hospital, 5 dropped out and others were not assessed the severity of depressive state by the author over 3 weeks after the beginning of treatment. Fifty-six patients ( 6 in-patients and 50 out-patients, 25 men and 31 women, aged $48.4 \pm 13.8$ years) were selected for data analysis. Of 56 patients, 7 were with melancholia, 12 with recurrence and 2 bipolar disorder. The mean HRSD total score was $16.4 \pm 5.6$. Five patients had first degree relatives with affective disorder. While the dose was originally designed as $150 \mathrm{mg}$ or $300 \mathrm{mg}$ per day, two patients received sulpiride $100 \mathrm{mg}$ per day voluntarily and one patient $450 \mathrm{mg}$ per day because of receiving other $150 \mathrm{mg}$ sulpiride from another hospital for gastrointestinal symptoms. Twenty-four patients received $150 \mathrm{mg}$ and 29 patients $300 \mathrm{mg}$ sulpiride per day. No significant demographic or psychopathological differences were found between the final and the excluded samples.

No significant relationship was obtained between the improvement rate (ratio of final to initial HRSD total score) and doses. GIR was as follows; 30 marked improvement, 13 improvement, 4 slight improvement, 4 no change, 2 slightly worse, 2 worse and one hypomanic change. Again there were no significant correlation between GIR and doses. Regarding doses per unit weight, the mean value was $4.6 \pm 1.9 \mathrm{mg}$ (range; $2.0-12.2 \mathrm{mg}$ ). No significant relationship was found between doses per unit weight and clinical response.

The plasma levels of sulpiride were analyzed for 44 patients. The mean value was $259 \pm 210 \mathrm{ng} / \mathrm{ml}$ (maximum $960 \mathrm{ng} / \mathrm{ml}$ ). The lower limit of detection was $20 \mathrm{ng} / \mathrm{ml}$. No significant relationship was found between the plasma levels and clinical effects.

The mean basal MHPG and DBH levels were $5.5 \pm 1.7 \mathrm{ng} / \mathrm{ml}$ and $31.5 \pm 16.9$ IU/L respectively. The mean post-treatment MHPG and DBH levels were $5.1 \pm 1.2$ $\mathrm{ng} / \mathrm{ml}$ and $30.5 \pm 14.4 \mathrm{IU} / \mathrm{L}$ respectively. No significant differences were found between pre-treatment and post-treatment MHPG or DBH levels. Neither MHPG nor DBH levels could predict clinical response.

The mean basal and post-treatment prolactin levels were $10.3 \pm 5.3$ and $112.8 \pm$ $87.2 \mathrm{ng} / \mathrm{ml}$ respectively. There was no relation between the post-treatment prolactin levels and plasma sulpiride levels. 
There were no differences of clinical effect with regard to melancholia by DSM III, New Castle Scale Diagnostic and ECT Indices, recurrence or heredity.

Two cases of Parkinsonism were observed during treatment. Their plasma levels of sulpiride were high; 540 and $960 \mathrm{ng} / \mathrm{ml}$.

\section{Discussion}

The present study failed to find any relationship between sulpiride plasma levels and clinical response. Salminen et al. ${ }^{2}$ studied 14 depressed patients with $150 \mathrm{mg}$ or $300 \mathrm{mg}$ sulpiride and failed to find the correlation between the blood levels and clinical response. Benkert et $a .^{3}$ administered $150 \mathrm{mg}$ sulpiride to 11 endogenously depressed patients and could not find any relationship between the blood levels and clinical effect.

According to an American Psychiatric Association task force report, ${ }^{10}$ some pitfalls should be considered when discussing issues related to blood levels of antidepressant. Firstly the measurement involves sophisticated technology. At present HPLC, used in the present study, is generally considered to be the most reliable and specific. A second critical issue is the patient population examined. Most investigators examined depressed in-patients. Concerning the compliance, in-patients are regarded more suitable. As pointed by this task force, however, out-patients studies may yield somewhat different information. Since the number of depressed out-patients are far greater than that of inpatients, results obtained from out-patients study are thought to be more valid for clinicians.

MHPG and DBH levels could not predict clinical rerponse in this study. Schildkraut $^{4}$ found that high MHPG predicted the effect of amitriptyline. Maas et al. ${ }^{5}$ reported that low MHPG levels had a significant correlation with the response of imipramine.

In the present study there was no relation between the prolactin levels and the sulpiride levels, while Bjerkenstedt et al. ${ }^{7}$ found the positive and significant correlation between the two levels. Hyperprolactinemia accompanied with sulpiride is generally thought to be caused by antidoparminagic action. Parkinsonism is another secondary effect of sulpiride by the same pharmacological action. Two cases, accompanied with parkinsonism, showed high plasma levels of sulpiride, which suggests that higher doses might possess more secondary effects.

\section{Acknowledgement}

The author wishes to thank Professor H. Hosaki and Lecturer K. Yagi for their supervision.

This study was supported by a grant from Sumitomo Pharmaceuticals Co. Ltd. 


\section{References}

1. Jenner, P. and Marsden, C. D.: The mode of action of sulpiride as an atypical antidepressant agent. Advances in Biochemical Psychopharmacology 32: 85-103, 1982

2. Salminen, J. K., Lehtonen, V., Allonen, H. and Kanto, J.: Sulpiride in depression: plasma levels and effects. Curr. Therap. Res. 27: 109-115, 1980

3. Benkert, O. and Holsboer, F.: Effect of sulpiride in endogenous depression. Acta Psychiatr. Scand. Suppl. 69: 43-48, 1984

4. Schildkraut, J. J.: Norepinephrine metabolites as biochemical criteria for classifying depressive disorders and predictive response to treatment. Preliminary findings. Am. J. Psychiatry 160: 695-698, 1973

5. Maas, J. W., Fawcett, J. A. and Dekirmenjian, H.: Catecholamine metabolism, depressive illness and drug response. Arch. Gen. Psychiatry 26: 252-262, 1972

6. Carney, M. W.P., Roth, M. and Garside, R. F.: The diagnosis and depressive syndromes and the prediction of E. C. T. response. Br. J. Psychiatry 111: 659-674, 1965

7. Bjerkenstedt, L., Härnryd, C. and Sedval, G.: Effect of sulpiride on monoaminergic mechanisms in psychotic women. Psychopharmacology 64: 135-139, 1979

8. Minegishi, A. and Ishizaki, T.: Rapid and simple method for the simultaneous determination of 3,4-dihydroxyphenylacetic acid, 5-hydroxyindole-3-acetic acid and 4-hydroxy3-methoxyphenylacetic acid in human plasma by high-performance liquid chromatography with electrochemical detection. J. Chromatogr. 308: 55-63, 1984

9. Nagatsu, T. and Udenfriend, S.: Photometric assay of dopamine- $\beta$-hydroxylase activity in human blood. Clin. Chem. 18: 980-983, 1972

10. Tricyclic antidepressant-blood level measurements and clinical outcome: an APA task force report. Am. J. Psychiatry 142: 155-162, 1985 